\title{
Energy Efficient NDMA Multi-packet Detection with Multiple Power Levels
}

\author{
Francisco Ganhão ${ }^{1,3}$, Miguel Pereira ${ }^{1,3}$, Luis Bernardo ${ }^{1}$, Rui Dinis ${ }^{1,3}$, \\ Rodolfo Oliveira ${ }^{1}$, Paulo Pinto ${ }^{1}$, Mário Macedo ${ }^{2,4}$, and Paulo Pereira ${ }^{4,5}$ \\ ${ }^{1}$ CTS, Uninova, Dep. de Eng. Electrotécnica, Faculdade de Ciências e Tecnologia, FCT, \\ Universidade Nova de Lisboa, 2829-516 Caparica, Portugal \\ ${ }^{2}$ Dep. de Eng. Electrotécnica, Faculdade de Ciências e Tecnologia, FCT, \\ Universidade Nova de Lisboa, 2829-516 Caparica, Portugal \\ ${ }^{3}$ IT, Instituto de Telecomunicações, Portugal \\ ${ }^{4}$ INESC-ID, Rua Alves Redol, 9. 1000-029 Lisboa, Portugal \\ ${ }^{5}$ Instituto Superior Técnico, Av. Rovisco Pais. 1049-001 Lisboa, Portugal \\ fjs.ganhao@gmail.com, miguelpereira.pro@gmail.com,

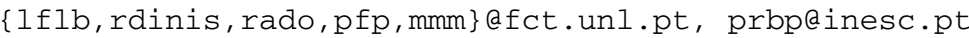

\begin{abstract}
Multi-packet detection approaches handle packet collisions and errors by forcing packet retransmission and by processing the resulting signals. NDMA (Network Diversity Multiple Access) detection approach forces $Q$ retransmissions by all stations when $Q$ stations transmit in one collision slot. Previous work assumed that perfect power control is used, where the average reception power is equal for all stations. In this paper we handle the scenario where no power control is used, and multiple power levels are received. We propose a modification to the basic NDMA (Network Diversity Multiple Access) reception mechanism, where some of the stations may not retransmit its packets all the times.

This paper evaluates the EPUP (energy per useful packet) and the goodput for a saturated uplink. Our analytical results are validated by simulation using joint PHY (physical layer) and MAC (Medium Access Control) behavior. We show that by suppressing some retransmissions we are able to improve the system's EPUP, practically without degrading the network goodput.
\end{abstract}

Keywords: multi-packet detection, NDMA, energy-per-useful-packet, goodput.

\section{Introduction}

The conventional approach to cope with collisions in a wireless channel is to discard all packets involved in the collision and to retransmit them. Multi-packet detection mechanisms use the signals associated to multiple collisions to separate the packets involved. In [1], a multi-packet detection technique was proposed, where all users involved in a collision of $Q$ packets retransmit their packets $Q-1$ times. The medium access control (MAC) protocol proposed was named network diversity multiple access (NDMA). To allow packet separation different phase rotations are employed for different packet retransmissions. An important drawback of the technique of [1] is 
that it is only suitable for flat-fading channels. Due to the linear nature of the receivers of [1], the residual interference levels can be high and/or can have significant noise enhancement. In [2] we proposed a frequency-domain multi-packet receiver that allows an efficient packet separation in the presence of successive collisions for NDMA. This receiver is suitable to severely time dispersive channels and does not require uncorrelated channels for different retransmissions. In [3] we extended NDMA to handle the situation where the multi-packet receiver is not able to handle a collision with all active Mobile Terminals (MTs). Yu et al. [4] proposed SICTA, an alternative approach that combines successive interference cancelation (SIC) with a tree algorithm (TA). In SICTA the collided packet signals are used to extract the individual packets, assuming a fixed flat-fading channel. In all cases, it was assumed perfect power control, i.e. equal average power level received at the base station (BS) from all users. However, the power of the received signal from a MT near the BS can be much higher than from the MTs further away of the BS.

This paper studies the goodput and the energy efficiency of NDMA when the BS receives different average power levels at the receiver BS from each user. It analyses the average energy used in the transmission of a successfully received packet for each MT $i$, i.e. the energy-per-useful-packet of MT $i\left(E P U P_{i}\right)$. The paper shows that energy efficiency can be improved if the users whose received signals are more powerfull do not retransmit all times, without degrading the saturation throughput and the EPUP for the remaining users. The system overview, including the packet detection and the MAC protocol proposed are presented in sec. 2. The systems' performance are analyzed in sec. 3 and a set of performance results is presented in sec. 4. Finally, sec. 5 is concerned with the conclusions of this paper.

\section{Contribution to Sustainability}

Energy efficiency is an emerging topic concerning the sustainability of a wireless communication. As a contribution to this topic, the research here presented applies an alternative medium access scheme to NDMA, assuming a non perfect power control, as opposed to other research works. With this scheme, the network's goodput does not degrade, maintaining a good energy efficiency.

\section{System Overview}

In this paper we consider the saturated uplink transmission in structured wireless systems employing Single Carrier with Frequency-Domain Equalization (SC-FDE) schemes, where a set of MTs send data to a BS. MTs are low resource battery operated devices whereas the BS is a high resource device, which runs the multipacket detection algorithm in real-time. MTs have a full-duplex radio and employ the NDMA algorithm to send data frames using the time slots defined by the BS (for the sake of simplicity, it is assumed that the packets associated to each user have the same number of bytes, $L_{\text {data }}$ ). The BS uses the downlink channel to acknowledge transmissions and, possibly, to force packet retransmissions or block the transmission of new packets in the next slot. It is assumed that different data frames arrive 
simultaneously and that perfect channel estimation, user detection, and synchronization between local oscillators exists. Data packets are composed of $N_{F F T}$ Fast Fourier Transform (FFT) blocks and have a physical preamble overhead of $N_{\text {PhyPreamble }}$ symbols. Each FFT block carries $N_{\text {Block }}$ symbols. The physical preamble is used to estimate the channel, synchronize the reception and detect the users involved in a given collision.

\subsection{Receiver Structure}

To achieve the separation of multiple data frames involved in a collision, we need to have multiple versions of each data frame involved in a collision. Classical NDMA requires $Q$ copies when $Q$ data frames are involved in a collision, but less copies are required when SIC is combined with NDMA. When $Q$ data frames are involved in a collision, the BS forces each MT to retransmit its frame up to $Q-1$ times. Let's assume that the packets are ordered by the reception power, such that $P_{q}^{r} \geq P_{q+1}^{r}$. The MT $q$ retransmits its packet (the $q$ th packet) $N_{q}$ times, where $q-1 \leq N_{q} \leq Q-1 . N_{q}$ is defined by the BS. Therefore, the receiver has $N_{q}+1$ versions of the signal of the $q$ th packet, and jointly detects all frames involved. We consider an iterative receiver that jointly performs the equalization and multipacket detection procedures, where each iteration consists of $Q$ detection stages, one for each frame.

When detecting a given packet we remove the residual interference from the other packets. For the detection of the $q$ th packet and the $i$ th iteration we use $Q$ frequencydomain feedforward filters, each one associated to the signal of a given collision (i.e., one retransmission), and $Q$ frequency-domain feedback filters, each one using the average value of the data signal associated to each packet.

The $k$ th frequency-domain sample associated to the $q$ th packet is $\widetilde{A}_{k, q}=\sum_{r=1}^{Q} F_{k, q}^{(r)} Y_{k}^{(r)}-\sum_{q^{\prime}=1}^{Q} B_{k, q}^{\left(q^{\prime}\right)} \bar{A}_{k, q^{\prime}}$. The average values $\bar{A}_{k, q^{\prime}}$ are obtained as follows. The block $\left\{\bar{A}_{k, q^{\prime}} ; k=0,1, \ldots, N-1\right\}$ is the discrete Fourier transform (DFT) of the block $\left\{\bar{a}_{n, q} ; n=0,1, \ldots, N-1\right\}$, where $\bar{a}_{n, q}$ denotes the average symbol values conditioned to the FDE output that can be computed as in [5]. It is shown in [2] that the optimal feedforward and feedback coefficients (selected to minimize the 'signalto-noise plus interference ratio', for a given packet and a given iteration) are given by $F_{k, q}^{(r, i)}=\breve{F}_{k, q}^{(r, i)} /_{\gamma_{q}^{(i)}}$, with $\gamma_{q}^{(i)}=\frac{1}{N} \sum_{k=0}^{N-1} \sum_{r=1}^{Q} \breve{F}_{k, q}^{(r, i)} H_{k, q}^{(r)}$, and $\breve{F}_{k, q}^{(r, i)}$ obtained from the set of $Q$ equations:

$$
\begin{gathered}
\left(1-\left|\rho_{q}^{(i)}\right|^{2}\right) H_{k, q}^{(r)^{*}} \sum_{r^{\prime}=1}^{Q} \breve{F}_{k, q}^{\left(r^{\prime}, i\right)} \breve{F}_{k, q}^{\left(r^{\prime}, i\right)}+ \\
\sum_{q^{\prime} \neq q}\left(1-\left|\rho_{q^{\prime}}^{(i)}\right|^{2}\right) H_{k, q^{\prime}}^{(r)^{*}} \sum_{r^{\prime}=1}^{Q} \breve{F}_{k, q}^{\left(r^{\prime}, i\right)} H_{k, q^{\prime}}^{\left(r^{\prime}\right)}+\alpha \breve{F}_{k, q}^{(r, i)}==H_{k, q}^{(r)^{*}}, r=1,2, \ldots, Q
\end{gathered}
$$

The feedback coefficients are then given by $B_{k, q}^{\left(q^{\prime}, i\right)}=\sum_{r=1}^{Q} F_{k, q}^{(r)} H_{k, q^{\prime}}^{(r, i)}-\delta_{q, q^{\prime}}\left(\delta_{q, q^{\prime}}=1\right.$ if $q=q$ ' and 0 otherwise). Clearly, the $Q$ feedback coefficients are used to remove 
interference between packets (as well as residual inter-symbol interference for the packet that is being detected). The feedforward coefficients are selected to minimize the overall noise plus the residual interference due to the fact that we do not have exact data estimates in the feedback loop. For high Signal-to-noise ratios (SNR) and when we do not have any information about the data (i.e., when $\rho_{q}=0$ ), the system of equations (1) gives the $F_{k, q}^{(r, i)}$ coefficients required to orthogonalize the other packets when detecting the $q$ th packet.

The system of equations inherent to (1) might not have a solution or it can be ill conditioned if the correlation between channels associated to different retransmissions is high. If the channel changes significantly from retransmission to retransmission (e.g. if different frequency channels are used for different retransmissions) this correlation can be very low. For systems where this is not practical, we consider the method proposed in [2] where the frequency domain block associated to the $r$ th retransmission of the $q$ th packet, $\left\{A_{k, q}^{(r)} ; k=0,1, \ldots, N-1\right\}$, is a cyclic-shifted version of $\left\{A_{k, q} ; k=0,1, \ldots, N-1\right\}$, with shift $\zeta_{r}$. In this paper we assume that the different $\zeta_{r}$ are the odd multiples of $N / 2, N / 4, N / 8, \ldots$ [2]. For severely time-dispersive channels this allows a small correlation between different $H_{k, q}^{(r)}$, for each frequency (naturally, as we increase $r$ we increase the correlations). Moreover, envelope fluctuations on the time-domain signal associated to $\left\{a_{n, q}^{(r)} ; n=0,1, \ldots, N-1\right\}$ are not too different from the ones associated to $\left\{a_{n, q} ; n=0,1, \ldots, N-1\right\}$.

\subsection{MAC Protocol}

The proposed MAC protocol follows the basic full-duplex NDMA approach [1][2]. The uplink slots are organized as a sequence of collision resolution periods, known as epochs. At the beginning of the epoch, any MT with data frames transmits. No new MTs are allowed to contend until the end of the epoch. An epoch lasts a number of slots equal to the number of MTs transmitting packets. When more than one station transmits, the BS uses the downlink channel to transmit an ACK frame at the end of the first slot, forcing the MTs to retransmit the data frames.

The basic NDMA only requires one bit to force the $Q-1$ retransmissions for every MT. In order to control the number of indivual retransmissions, the ACK frame must include the list of MAC addresses detected, and the array with the $N_{q}$ values, which specify the minimum number of retransmissions required for each colliding MT. Failed data frames are retransmitted in the next epochs up to $M_{R}$ times before being dropped. In the following analysis we do not take into account the duration of the ACK frame, which is transmitted between data frames.

\section{Performance Analysis}

\subsection{Goodput Analysis}

The MultiPacket Reception (MPR) system can be characterized by the probability of a MT $l$ successfully transmitting a packet, given by $q_{Q, l}, 0 \leq q_{Q, l} \leq 1$, with $l, 1 \leq l \leq Q$, and 
where $Q$ defines the number of MTs colliding. This probability is system specific, and depends on the successful reception of every bit, $b e r_{Q, l}$, which is influenced by the set of received powers at the BS and the $N_{q}$ values. Assuming a fixed size of $L_{\text {data }}$ bits per packet, then $q_{Q, l}$ is given by

$$
q_{Q, l}=\left(1-\operatorname{ber}_{Q, l}\right)^{L_{d a t a}}
$$

Let $S_{l}^{\text {sat }}$ be the saturation goodput for MT $l$. It can be calculated by the ratio of the expected number of packets successfuly transmitted by MT $l$ (with up to $R-1$ retransmissions) to the expected packet transmission duration, and is given by

$$
\begin{aligned}
S_{l}^{s a t}= & \frac{\sum_{r=1}^{R} q_{Q, l}\left(1-q_{Q, l}\right)^{r-1}}{Q R\left(1-q_{Q, l}\right)^{R}+\sum_{r=1}^{R} Q r q_{Q, l}\left(1-q_{Q, l}\right)^{r-1}} \\
= & \frac{q_{Q, l}\left(1-\left(1-q_{Q, l}\right)^{R}\right)}{q_{Q, l} Q R\left(1-q_{Q, l}\right)^{R}+Q\left(1-\left(1-q_{Q, l}\right)^{R}\right)^{2}} .
\end{aligned}
$$

The total channel's saturation goodput is,

$$
S^{s a t}=\sum_{l=1}^{Q} S_{l}^{s a t} .
$$

\subsection{Energy Analysis}

For a low power transmission system, the energy consumption model has to consider both the transmission energy and the circuit energy consumption. Cui et al. [6] proposed an energy model for uncoded M-QAM (M-ary quadrature amplitude modulation) that can be adapted to our scenario, considering both, the transmission and reception energy consumption for full-duplex, during the slot time, $T_{\text {slot }}$. Cui et al. show that the energy consumption per packet transmission in MT $l$ is approximately given by $E_{l}^{p} \approx(1+\beta) P_{l}^{t} T_{\text {slot }}+P_{c} T_{\text {slot }}$, where $P_{c}$ denotes the total circuit energy consumption and $\beta=P_{\text {amp }} / P_{l}^{t}$ is the ratio of power consumption on the power amplifier to the transmitted power $\left(P_{l}^{t}\right)$. This ratio is given by $\beta=\xi / \eta-1$ with $\eta$ as the drain efficiency of the radio frequency power amplifier and $\xi$ as the peak-to-average-ratio.

An uncoded scenario with one bit per symbol was considered for this paper due to its extreme simplicity. Thus, $\xi=\frac{\max \left(b_{n} b_{n}^{*}\right)}{\left.E\left[b_{n} b_{n}^{*}\right]\right]}=1$. Assuming the $\kappa$ th-power path-loss model at distance $d$ (meters), the transmission power is expressed as $P_{t}=P_{r} G_{1} d^{\kappa} M_{l}$, where $P_{r}$ is the received power, $M_{l}$ is the link margin compensating 
the hardware process variations and other additive noise, and $G_{l}$ is the gain factor at $d=1 \mathrm{~m}$. An AWGN power spectral density of $\sigma^{2}=N_{0} / 2=-174 \mathrm{dBm} / \mathrm{Hz}$ was considered for a given bandwidth $B$.

Assuming $P_{r}=M E_{b} / T_{\text {slot }}$, the expended energy for each packet is defined as

$$
E_{l}^{p} \approx(1+\beta) G_{1} d^{\kappa} M_{l} M E_{b}+P_{c} T_{o n}
$$

The energy per useful packet of MT $l$, denoted $E P U P_{l}$, measures the average transmitted energy for a correctly received packet. It is given by:

$$
\begin{aligned}
E P U P_{l} & =\frac{E_{l}^{p} R\left(1-q_{Q, l}\right)^{R}+\sum_{r=1}^{R} E_{l}^{p} r q_{Q, l}\left(1-q_{Q, l}\right)^{r-1}}{1-\left(1-q_{Q, l}\right)^{R}} \\
& =E_{l}^{p}\left(\frac{R\left(1-q_{Q, l}\right)^{R}}{1-\left(1-q_{Q, l}\right)^{R}}+\frac{1-\left(1-q_{Q, l}\right)^{R}}{q_{Q, l}}\right) .
\end{aligned}
$$

Therefore, the channel's average EPUP, is

$$
E P U P=\left(\sum_{l=1}^{Q} E P U P_{l}\right) / Q .
$$

\section{Simulations}

The current section presents a performance analysis of the proposed system model for a flat channel, assuming a saturated system. The results were measured using Monte Carlo simulations. Table 1 presents the energy model parameters.

The results hereof, assume two groups of nodes: A with $\mathrm{Q}_{1}$ MTs and B with $\mathrm{Q}_{2}$. The former group transmits with maximum transmission power, while the latter transmits with $40 \%$. A base station receives packets from both groups and manages the scheduling of collided packets. A packet collision, might include nodes from both groups or just one. The receiver's structure supports up to $\mathrm{Q}=4$ collisions. For simplicity purposes, it is assumed that at most one node from group A, transmits at the same time with up to Q-1 nodes from group B. Nodes are spatially distributed around the base station at a distance of $20 \mathrm{~m}$.

Table 1. Simulation parameters

\begin{tabular}{cccccc}
\hline Parameter & Value & Parameter & Value & Parameter & Value \\
\hline$P_{c}$ & $164.998 \mathrm{~mW}$ & $\xi$ & 1 & $\eta$ & 0.35 \\
$T_{\text {slot }}$ & $0.0205 \mathrm{~ms}$ & $B$ & $2.5 \mathrm{MHz}$ & $L_{\text {data }}$ & $1024 \mathrm{bit}$ \\
$G_{1}$ & $30 \mathrm{~dB}$ & $\kappa$ & 3.5 & $M_{\iota}$ & $40 \mathrm{~dB}$ \\
\hline
\end{tabular}


Figure 1, presents the bit error rate (BER) of an AWGN channel. It shows that the BER improves for an increasing number of collisions (higher Q). The BER reduction is more significant when the interfering signals have lower power. Therefore, the proposed iteractive receiver is capable of removing interference with multiple receiver power levels.

Figure 2 illustrates the energy per useful packet (EPUP). For a fixed number of $\mathrm{Q}=4$ collisions, it is observable that an increasing number of retransmissions for each epoch, degrade the EPUP for a saturated channel, the same applies for nodes of a weaker transmission power. Correlating the EPUP with the goodput from Figure 3, it is conclusive that the optimum, or minimum, EPUP is obtained once the goodput reaches its maximum. The goodput is also affected for nodes with a weaker transmission power.

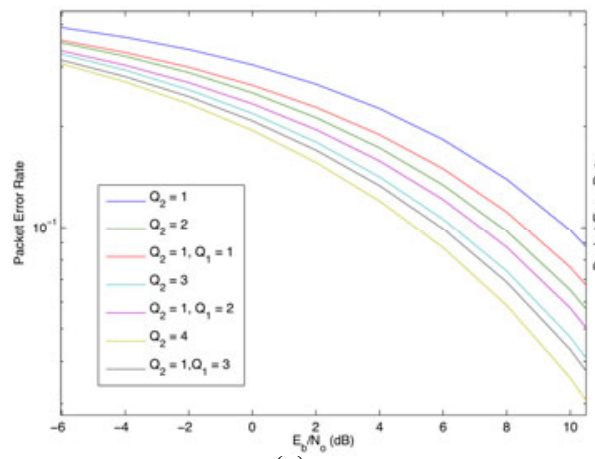

(a)

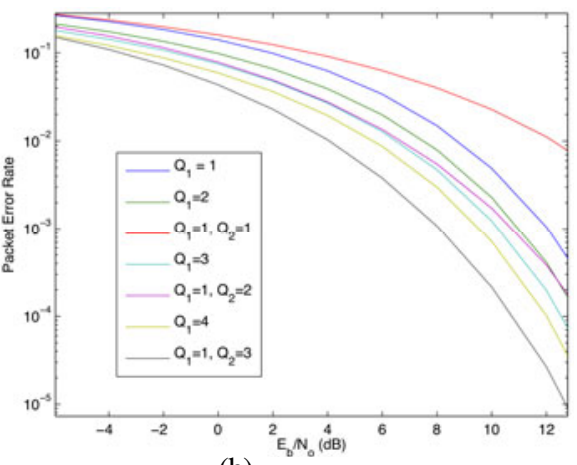

(b)

Fig. 1. Bit error rate: (a) $\mathrm{Q}_{2}$ nodes that transmit with $40 \%$ of the maximum transmission power; (2) $\mathrm{Q}_{1}$ nodes that transmit with $100 \%$ of the maximum transmission power

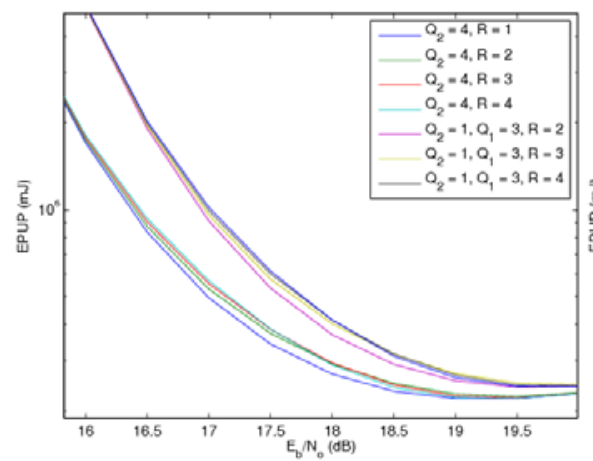

(a)

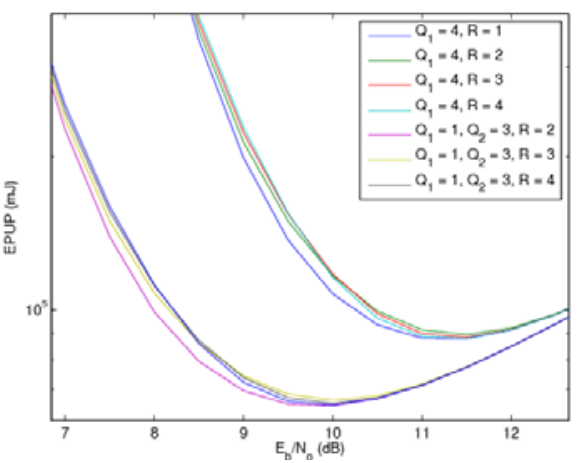

(b)

Fig. 2. Energy per useful packet: (a) $\mathrm{Q}_{2}$ nodes that transmit with $40 \%$ of the maximum transmission power; (2) $\mathrm{Q}_{1}$ nodes that transmit with $100 \%$ of the maximum transmission power 


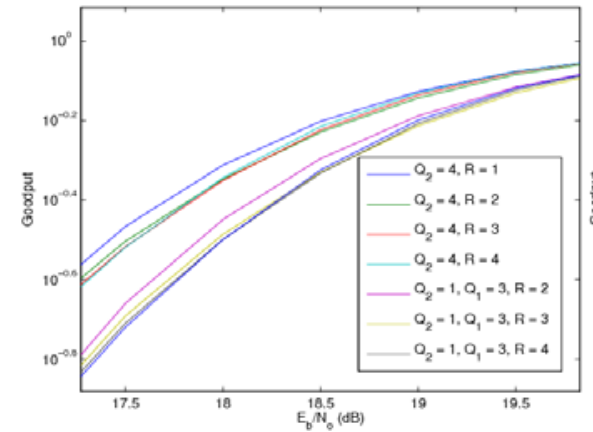

(a)

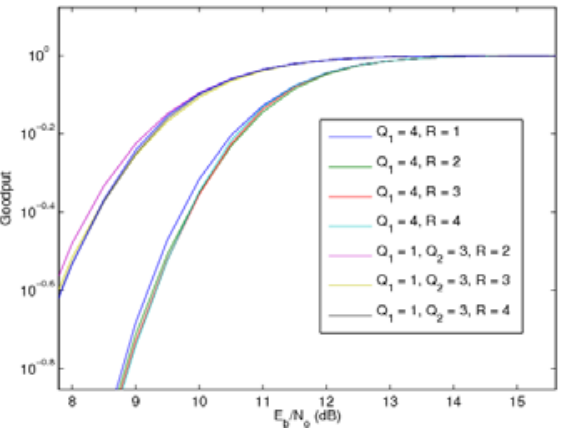

(b)

Fig. 3. Goodput: (a) $\mathrm{Q}_{2}$ nodes that transmit with $40 \%$ of the maximum transmission power; (2) $\mathrm{Q}_{1}$ nodes that transmit with $100 \%$ of the maximum transmission power

\section{Conclusions}

In this paper, we have proposed a new approach to handle differentiated power levels at an MPR receiver and analysed the resulting energy efficiency and goodput. The paper shows that the suppression of retransmissions of the most powerful signals increases the system goodput and reduces the EPUP, showing that this approach allows a performance improvement of NDMA over classic NDMA with perfect power control. For future research, it is intended to enhance the analytical model for an unsaturated channel and study its performance with upper layer protocols. Other research options are also available, such as enhancing the proposed architecture in terms of scheduling and the receiver's structure.

\section{References}

1. Tsatsanis, M.K., Ruifeng, Z., Banerjee, S.: Network-assisted diversity for random access wireless networks. IEEE Transactions on Signal Processing 48(3), 702-711 (2000)

2. Dinis, R., Montezuma, P., Bernardo, L., Oliveira, R., Pereira, M., Pinto, P.: Frequencydomain multipacket detection: a high throughput technique for SC-FDE systems. IEEE Trans. on Wireless Communications 8(7), 3798-3807 (2009)

3. Pereira, M., Bernardo, L., Dinis, R., Oliveira, R., Carvalho, P., Pinto, P.: A MAC Protocol for Half-Duplex Multi-Packet Detection in SC-FDE Systems. In: IEEE Vehicular Technology Conference (VTC)-Spring, IEEE Press, Barcelona (2009)

4. Yu, Y., Giannakis, G.B.: SICTA: a 0.693 contention tree algorithm using successive interference cancellation. IEEE/ACM Trans. on Networking 3, 1908-1916 (2005)

5. Dinis, R., Carvalho, P., Martins, J.: Soft Combining ARQ Techniques for Wireless Systems Employing SC-FDE Schemes. In: 17th Int. Conf. on Computer Communications and Networks, pp. 1-5. IEEE Press, St. Thomas U.S. Virgin Islands (2008)

6. Cui, S., Goldsmith, A.J., Bahai, A.: Energy-constrained modulation optimization. IEEE Trans. on Wireless Communications 4(5), 2349-2360 (2005)

7. NS-2 Network Simulator, version 2.34 (2009), http: / /www. isi . edu/nsnam/ns / 